\section{Multiplex Allele-Specific PCR with Optimized Locked Nucleic Acid Primers}

BioTechniques 34:1150-1158 (June 2003)

The ease and advantages of allelespecific PCR (AS-PCR) for SNP genotyping, along with the difficulty of assay specificity with certain mismatched DNA primers, have been described in prior studies (1-3). More recently, the use of real-time PCR detection formats has been described for one or both alleles of AS-PCR (4-6). These detection enhancement methods do not overcome the inherent difficulties with mismatch specificity and may also be limited by the choice of dyes or instrumentation for larger multiplex AS-PCR protocols. Researchers have attempted to utilize multiplex PCR in allele-specific applications $(7,8)$. These approaches add a level of complexity to the design and optimization of DNA primers, negating some of the ease and speed of the original AS-PCR technique.

Several molecular applications of locked nucleic acid (LNA) $(9,10)$ have been described in review articles $(11,12)$. These LNA applications include antisense and therapeutic techniques $(13,14)$, SNP genotyping by fluorescence polarization (15), and hybridization-based probe detection (16). Our prior work showed improved ASPCR specificity with $3^{\prime}$ LNA primers at the polymorphic position in SNP genotyping with human and plasmid DNA targets with all possible mismatches (17). LNA is believed to exert its effects of increased hybrid stability (higher $\mathrm{T}_{\mathrm{m}}$ ) and improved specificity in AS-PCR through the interaction of its rigid conformational structure with complementary strands or enzymes, resulting in slower rates of dissociation (18). In this report, SNPs within the human cystic fibrosis gene (CFTR) were analyzed in individual PCRs with 3' LNA and DNA primers under various reaction conditions. Once optimization was completed, multiplex PCR amplification reactions were investigated with individual and pooled templates and detected by gel analysis.

Table 1. CFTR Allele-Specific LNA and Companion Primer Pairs

\begin{tabular}{|c|c|c|c|}
\hline Mutation & WT or MT & Primer Sequence $\left(5^{\prime} \rightarrow 3^{\prime}\right)$ & $\begin{array}{l}\text { Product } \\
\text { Size (bp) }\end{array}$ \\
\hline G551D & WT & gctaaagaaattcttgctcgttgaC & 535 \\
\hline G551D & $\mathrm{MT}$ & gctaaagaaattcttgctcgttgaT & 535 \\
\hline G551D & na & aatgttcttttagtttctcttcttaggagg & 535 \\
\hline G551D & MT & gtggaatcacactgagtggagA & 277 \\
\hline G551D & na & tgacaccaagatacgggcacag & 277 \\
\hline A455E & MT & agatagaaagaggacagttgttggA & 454 \\
\hline A455E & na & tggagactttttgtttatgtggttac & 454 \\
\hline 3659delC & MT & cactgccaacagaaggtaaacctaA & 415 \\
\hline 3659delC & na & cccattatataggttcaggactctgc & 415 \\
\hline delF508 & MT & tggcaccattaaagaaaatatcatT & 400 \\
\hline delF508 & na & atgccatataaataaccattgaggacg & 400 \\
\hline R334W & MT & cactaatcaaaggaatcatcctcT & 346 \\
\hline R334W & na & gccactctcatccatcatactgt & 346 \\
\hline G542X & MT & ttgcagagaaagacaatatagttcttT & 311 \\
\hline G542X & na & tgacaccaagatacgggcacag & 311 \\
\hline R1162X & MT & tcagatgcgatctgtgagcT & 274 \\
\hline R1162X & na & gcaagcagtgttcaaatctcacc & 274 \\
\hline 1717-1G>A & MT & ctctctaattttctatttttggtaataA & 221 \\
\hline 1717-1G>A & na & ccagaaacagaatataaagcaatagaga & 221 \\
\hline
\end{tabular}

AS-PCR primer sequences (with LNA bases in capital letters) are listed along with companions for each pair. The expected PCR product sizes from each primer pair are shown. For the G551D polymorphism, the antisense strand was targeted in Figure 1 and the sense strand in Figure 2. WT, wild-type; MT, mutant.

LNA primers demonstrated higher specificity than DNA primers in multiplex AS-PCR with human SNP targets. This strong allelic discrimination with LNA primers was evident over a broad range of parameters, demonstrating a simplified approach to highly specific multiplex AS-PCR.

All heterozygous human DNA samples containing mutant CFTR alleles were purchased from the Coriell Cell Repository (Camden, NJ, USA). Wildtype human genomic DNA was purchased from Promega (Madison, WI, USA) and verified for each lot. All PCR primers were produced by Proligo (Boulder, CO, USA) and are listed in Table 1 with either $3^{\prime}$ LNA or native DNA residues. PCR variables for Figures 1 and 2 are listed in Table 2. These include concentrations of $\mathrm{MgCl}_{2}$, primer, AmpliTaq Gold ${ }^{\circledR}$ DNA polymerase (Applied Biosystems, Foster City, CA, USA), and template DNA. Constant PCR parameters were $1 \times$ Gold reaction buffer (Applied Biosystems),
dNTP concentration $(200 \mu \mathrm{M})$, reaction volume $(25 \mu \mathrm{L})$, and thermal cycling using a DNA Engine ${ }^{\mathrm{TM}}$ (MJ Research, Waltham, MA, USA): $95^{\circ} \mathrm{C}$ for $7 \mathrm{~min}$, followed by 30 cycles of $94^{\circ} \mathrm{C}$ for $30 \mathrm{~s}$, $61^{\circ} \mathrm{C}$ for $30 \mathrm{~s}$, and $72^{\circ} \mathrm{C}$ for $30 \mathrm{~s}$.

AS-PCR optimization was done with 3' LNA and DNA primers in more than 20 target SNPs in three human genes (CFTR, adenosine deaminase, and $\mathrm{T}$ cell receptor $\mathrm{V} \beta$ ). LNA-containing primers required no difference in preparation or significant changes in reaction and thermal cycling parameters compared with DNA primers. Figure 1 is a comparison of LNA primers with DNA primers for detection of a cystic fibrosis G551D mutation $(\mathrm{G} \rightarrow \mathrm{A})$ in wild-type and heterozygous human genomic DNA samples under five PCR conditions (labeled A-E) listed in Table 2.

Wild-type DNA template was used to assess whether mutant AS-PCR primers produced false-positive products in the absence of a mutant allele. In each panel, LNA mutant primers 


\section{Benchmarks}

Table 2. PCR Conditions for Experiments Shown in Figures 1 and 2

\begin{tabular}{|ccccc|}
\hline Condition & $\begin{array}{c}\mathrm{MgCl}_{2} \\
(\mathbf{m M})\end{array}$ & $\begin{array}{c}\text { Primer } \\
(\mu \mathrm{M})\end{array}$ & $\begin{array}{c}\text { Taq } \\
(\mathbf{U})\end{array}$ & $\begin{array}{c}\text { gDNA } \\
(\mathbf{n g})\end{array}$ \\
\hline $\mathrm{A}$ & 2 & 0.10 & 1.0 & 10 \\
$\mathrm{~B}$ & 2 & 0.25 & 1.0 & 10 \\
$\mathrm{C}$ & 4 & 0.10 & 1.0 & 10 \\
$\mathrm{D}$ & 4 & 0.25 & 1.0 & 10 \\
$\mathrm{E}$ & 6 & 0.50 & 1.0 & 20 \\
Figure 2 & 8 & 0.10 & 1.5 & 20
\end{tabular}

Differences in reaction conditions for conditions A-E in Figure 1 and the single condition in Figure 2 are listed.

discriminated better than DNA mutant primers by not producing a false band in lane 4 (versus lane 3 ) with wild-type genomic DNA samples. This same LNA mutant primer functioned well when given the correct mutant target (lane 8 of each set). The difference in band intensities between mismatched LNA and DNA primers was greatest in conditions C-E (with greater than $2 \mathrm{mM} \mathrm{MgCl}_{2}$ ) in Figure 1. These titration experiments showed that LNA primers exhibited allelic specificity over a broad range of conditions [e.g., 2-6 $\mathrm{mM} \mathrm{MgCl}_{2}$, $0.1-0.5 \mu \mathrm{M}$ primer, and $35-200 \quad \mu \mathrm{M}$ dNTPs (data not shown)], while DNA primers often failed to discriminate over these same ranges.

It was observed that $3^{\prime}$ LNA primers successfully identified all transition and transversion SNPs queried. The fact that LNA primers maintained proper allelic discrimination over a broad range of PCR parameters indicated that this LNA effect was not condition-sensitive. This can effectively reduce single and multiplex AS-PCR primer design and optimization time. In experiments testing many parameters with LNA and DNA primers for several SNPs, the best AS-PCR product yields occurred with condition E (6 $\mathrm{mM} \mathrm{MgCl} 2,20 \mathrm{ng}$ genomic DNA, and $0.5 \mu \mathrm{M}$ primers).

Multiplex AS-PCR results in Figure 2 used a primer pool consisting of eight CFTR mutant primers plus their counterpart primers listed in Table 1 . Human multiplex AS-PCR with DNA and LNA primer pools targeted the sense strands of eight mutant CFTR alleles and included 16 allele-specific and companion primers. We then challenged these primer pools to correctly identify the presence or absence of SNP mutant alleles in individual and pooled template DNA samples, as shown in Figure 2. The individual template samples produced the correct number and size of products with LNA primers (lanes 2 and 4) under the multiplex conditions used, whereas DNA primers generated additional nondiscriminatory products through mispriming (lanes 1 and 3). These misprimed DNA products can clearly be seen as wild-type CFTR alleles by visual match with the pooled mutant allele controls (lanes 6 and 7) described below.

As a control for the pooled mutant primers, two pooled template samples were interrogated. One template pool contained all eight mutant alleles (lanes 6 and 7), demonstrating that all LNA and DNA mutant primers amplify with comparable efficiency when their correctly matched alleles are present. The other template pool contained no mutations, showing a difference between LNA and DNA primers when their cognate alleles are not present (lanes 8
Figure 1. AS-PCR optimization of human CFTR polymorphism G551D with DNA and 3' LNA matched and mismatched primers. Four AS-PCR primers, all paired with a common companion, were tested under five different PCR conditions (A-E) with both wild-type and heterozygous mutant templates. Primers denoted DNA or 3' LNA wild-type (DW, LW) or mutant (DM, LM) are arranged in the same pattern in lanes 1-8 in each gel image. Genomic DNA samples were wild-type (WT) in lanes 1-4 and heterozygous mutant (MT) in lanes 5-8, as specified by the Coriell Institute. 


\section{Benchmarks}

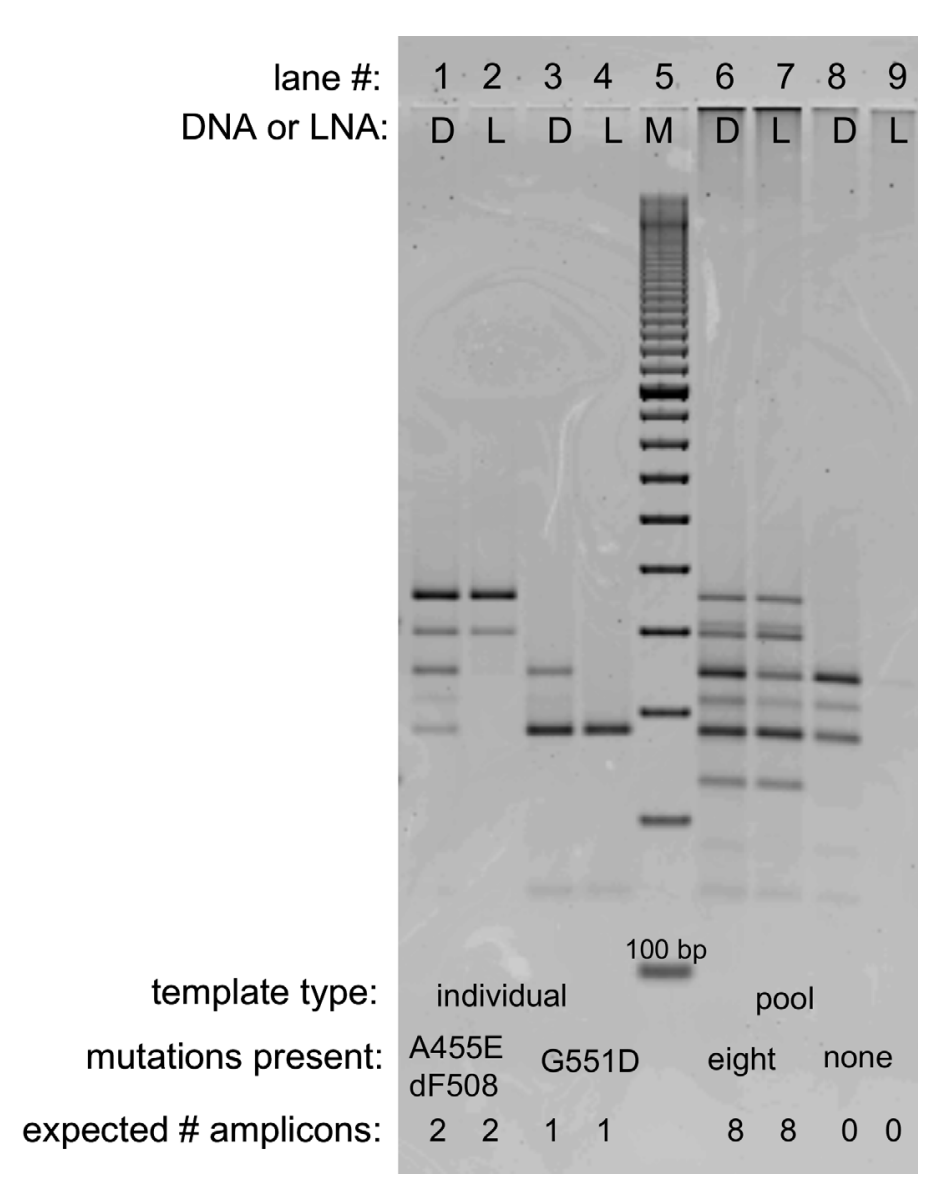

Figure 2. Multiplex AS-PCR detection of eight CFTR mutations with $3^{\prime}$ LNA and DNA primers. Image of a $2 \%$ agarose gel stained with ethidium bromide, showing DNA or $3^{\prime}$ LNA mutant type primers (D or L) with sequences listed in Table 1. The CFTR mutations (A455E, $\triangle \mathrm{F} 508$, and G551D) present in the individual templates are indicated. Individual template samples are shown in lanes $1-4$, while pooled genomic DNA samples both with (lanes 6 and 7) and without (lanes 8 and 9) SNP mutations are shown. A 100-bp marker ladder (M, Bio-Rad Laboratories, Hercules, CA, USA) is shown between the two sets. The expected number of PCR products/lane is indicated at the bottom.

and 9). Only the LNA primer pool exhibited proper PCR specificity by generating no products from the wild-type template pool (lane 9). The analogous DNA primer pool (lane 8) exhibited three false-positive bands, which correlate to mismatched products for G542X (T:C), R334W (T:G), G551D (A:C), and/or R1162W (T:G). The three falsepositive bands in lane 8 may represent three or four products, since two products nearly overlap for polymorphisms R1162X and G551D (274 and 277 bp). The limits of sensitivity and specificity of LNA and DNA primers were not examined beyond the 8-plex level in these experiments, so an upper limit has not been established.
In summary, the use of $3^{\prime}$ LNA-containing primers is a rapid and reliable method for obtaining highly accurate genotyping results using AS-PCR. The multiplex result highlights the specificity of LNA primers in genotype analysis of eight mutant CFTR alleles. The LNA primers yielded no false-positive products with individual or pooled template samples in this example, while DNA primers failed to correctly discriminate mismatches in each case. The three false-positive bands for DNA pooled mutant primers (lane 8 ) versus none for 3' LNA primers (lane 9) is a striking example of enhanced LNA allelic specificity and its potential in multiplex AS-PCR applications. Along with the simplified design, the wide window of effective conditions, and the baseline protocol established for multiplex AS-PCR with LNA primers, this technique offers strong potential in important applications such as haplotype analysis by long-range PCR.

\section{REFERENCES}

1.Huang, M.M., N. Arnheim, and M.F. Goodman. 1992. Extension of base mispairs by Taq DNA polymerase: implications for single nucleotide discrimination in PCR. Nucleic Acids Res. 20:4567-4573.

2.Newton, C.R., A. Graham, L.E. Heptinstall, S.J. Powell, C. Summers, N. Kalsheker, J.C. Smith, and A.F. Markham. 1989. Analysis of any point mutation in DNA. The amplification refractory mutation system (ARMS). Nucleic Acids Res. 17:2503-2516.

3.Ugozzoli, L. and B. Wallace. 1991. Allelespecific polymerase chain reaction. Methods 2:42-48

4.Moran, P., D.A. Dightman, and L.K. Park. 1998. Nonelectrophoretic genotyping using allele-specific PCR and a dsDNA-specific dye. BioTechniques 24:206-212.

5.Thorpe, P.H. and D.J. Porteous. 1999. Rapid quantitation of gene therapy specific CFTR expression using the amplification refractory mutation system. BioTechniques 27:122-127.

6.Waterfall, C.M. and B.D. Cobb. 2002. SNP genotyping using single-tube fluorescent bidirectional PCR. BioTechniques 33:80-90.

7.Broude, N.E., L. Zhang, K. Woodward, D. Englert, and C.R. Cantor. 2001. Multiplex allele-specific target amplification based on PCR suppression. Proc. Natl. Acad. Sci. USA 98:206-211.

8.Ferrie, R.M., M.J. Schwarz, N.H. Robertson, S. Vaudin, M. Super, G. Malone, and S. Little. 1992. Development, multiplexing and application of ARMS tests for common mutations in the CFTR gene. Am. J. Hum. Genet. 51:251-262.

9.Koshkin, A.A., S.K. Singh, P. Nielsen, V.K. Rajwanshi, R. Kumar, R.M. Meldgaard, C.E. Olsen, and J. Wengel. 1998. LNA (locked nucleic acids): synthesis of the adenine, cytosine, guanine, 5-methylcytosine, thymine and uracil bicyclonucleoside monomers, oligomerisation, and unprecedented nucleic acid recognition. Tetrahedron 54:3607-3630.

10.Obika, S., D. Nanbu, Y. Hari, J. Andoh, K. Morio, T. Doi, and T. Imanishi. 1998. Stability and structural features of the duplexes containing nucleoside analogues with a fixed $\mathrm{N}$ type conformation, 2'-O,4'-C-methylene ribonucleosides. Tetrahedron Lett. 39:54015404.

11.Braasch, D.A. and D.R. Corey. 2000. Locked nucleic acid (LNA): fine tuning the recognition of DNA and RNA. Chem. Biol. 55:1-7.

12.Mouritzen, P., A.T. Nielson, H.M. Pfundheller, Y. Choleva, L. Kongsbak, and S. Moller. 2003. SNP genotyping using LNA 


\section{Benchmarks}

(locked nucleic acid). Exp. Rev. Mol. Diagn. 3:7-38.

13.Kurreck, J., E. Wyszko, C. Gillen, and V.A. Erdmann. 2002. Design of antisense oligonucleotides stabilized by locked nucleic acids. Nucleic Acids Res. 30:1911-1918.

14.Petersen, M. and J. Wengel. 2003. LNA: a versatile tool for therapeutics and genomics. Trends Biotechnol. 21:74-81.

15.Simeonov, A. and T.T. Nikiforov. 2002. Single nucleotide polymorphism genotyping using short, fluorescently labelled locked nucleic acid (LNA) probes and fluorescence polarization detection. Nucleic Acids Res. 30:e91.

16.Orum, H., M.H. Jakobsen, T. Koch, J. Vuust, and M.B. Borre. 1999. Detection of the factor V Leiden mutation by direct allele-specific hybridization of PCR amplicons to photoimmobilized locked nucleic acids. Clin. Chem. 45:1898-1905.

17.Latorra, D., K. Campbell, A. Wolter, and J.M. Hurley. Enhanced allele specific PCR discrimination in SNP genotyping using 3 locked nucleic acid (LNA) primers. Hum. Mutat. (In Press.)

18.Christensen, U., N. Jacobsen, V.K. Rajwanshi, J. Wengel, and T. Koch. 2001. Stoppedflow kinetics of locked nucleic acid (LNA) oligonucleotide duplex formation: studies of LNA-DNA and DNA-DNA interactions. Biochem. J. 354:481-484.

Address correspondence to Dr. David Latorra, Proligo LLC, 6200 Lookout Road, Boulder, CO 80301, USA. e-mail: dlatorra@proligo.com

Received 27 February 2003; accepted 31 March 2003.

David Latorra, Deborah Hopkins, Krista Campbell, and J. Michael Hurley

Proligo LLC

Boulder, CO, USA

\section{Encapsulation of Acetylcholinesterase in Preformed Liposomes}

BioTechniques 34:1158-1162 (June 2003)

Acetylcholinesterase (AChE) is highly sensitive to pesticides and has been engineered to become a real tool for their detection (1). However, its utilization in biosensors faces a major problem. The enzyme is not stable enough to be useful for pesticide detection in the field. One way to increase an enzyme's stability is to encapsulate it in liposomes or nanocapsules $(2,3)$. This encapsulation stabilizes the enzyme against dilution effects and protects it against proteolytic agents. In the following experiments, we encapsulated $\mathrm{AChE}$ in liposomes. For encapsulation, we tried different established techniques. The first one forms the capsule from lipids in the presence of protein. The technique consists of mixing a protein-containing aqueous solution with a lipid solution solubilized either by a detergent or by an organic solvent. By removing either the detergent by dialysis (4) or the organic solvent by evaporation [the reverse-phase evaporation method (5)], the lipids organize in bilayers around aqueous droplets of enzyme solution and form liposomes. However, these techniques are highly damaging for fragile proteins that are denatured by detergents, solubilized lipids, or organic solvents. When we tested the detergent dialysis technique, we noticed that $50 \%$ of AChE involved in this process was denatured; $70 \%$ was denatured by the reverse-phase evaporation method (data not shown). An alternative technique consists of adding the protein to a preformed dry lipid film. The dispersion of the dry lipid film with a protein-containing aqueous solution leads to the formation of multilamellar vesicles. They are converted to monolamellar vesicles by repeated freeze-thaw cycles or by sonication $(6,7)$. During sonication, however, the intact membrane is lysed. These membrane fragments seem to behave like a detergent and denature the AChE up to $90 \%$ after $1 \mathrm{~min}$ of sonication (data not shown). Therefore, the most suitable 